\title{
El arte danzario como eje en la educación inclusiva
}

\section{The art of dance as an axis in inclusive education}

"La inclusión se caracteriza por una experiencia social ampliamente compartida y la participación activa de una sociedad, por la igualdad generalizada de las posibilidades y las oportunidades de la vida que se ofrecen a los individuos en el plano individual, y por el logro de un nivel de bienestar elemental para todos los ciudadanos." (Citado por UNESCO, 2008).

\section{Lina María Gámez Bolívar ${ }^{1}$}

\section{Astrid Ramírez Valencia ${ }^{2}$}

\section{Yury de Jesús Ferrer Franco ${ }^{3}$}

Universidad Distrital Francisco José de Caldas
$1 \quad$ Estudiante en formación del programa de Licenciatura en Educación Básica con Énfasis en Educación Artística, Universidad Distrital Francisco José de Caldas, Docente en formación en diferentes instituciones educativas de primaria y secundaria, privadas. Correo limgamezb@ correo.udistrital.edu.co. ORCID https://orcid.org/0000-00033728-7853

2 Docente-investigadora, Universidad Distrital Francisco José de Caldas Bogotá, Colombia, Ph.D. en Lenguaje y cultura, formadora de profesores de inglés durante más de 25 años. Investigadora y maestra en la Universidad Distrital Francisco José de Caldas. correo aramirezv@udistrital.edu.co ORCID: https://orcid.org/0000-0002-3025-5982 3 Docente-investigador, Universidad Distrital Francisco José de Caldas Bogotá, Colombia, Doctorando en Cultura y Educación en América Latina, Magíster en Literatura, Licenciado en Filología e Idiomas (español-francés), Comunicador Social -Periodista; profesor de lengua materna y literatura desde 1990. Correo electrónico ydferrerf@udistrital.edu.co ORCID: https://orcid.org/0000-0001-5015-5172

\section{RESUMEN}

En la actualidad, el empoderamiento de la educación inclusiva en Colombia, sigue enfrentándose a distintas líneas de acción y pensamientos, encaminadas a satisfacer las necesidades de discapacidad de cierta población con miras a generar acciones de inclusión en el aula de clase. El objetivo de este artículo es tomar en consideración diversas miradas tejidas alrededor de esta temática, con miras a reflexionar y considerar diversas posturas que permitan el planteamiento de una estrategia 
pedagógica sustentada en el arte de la danza, con la cual sea posible visualizar una percepción más amplia que ponga en marcha una nueva puesta de inclusión dentro del aula, se concluye, que la danza se constituye en un elemento fundamental que propende por el mejoramiento de las relaciones interpersonales integradoras de todos los agentes educativos que incluyen a los niños y niñas con discapacidad o no así como a sus cuidadores, por permitir el reconocimiento, la empatía de sí mismo y la aceptación del otro. sin ningún tipo de discriminación.

\section{PALABRAS CLAVE:}

Educación incluyente- danza, cuidadores

\section{ABSTRACT}

Currently, the empowerment of inclusive education in Colombia continues to face different lines of action and thoughts, aimed at meeting the disability needs of a certain population with a view to generating inclusive actions in the classroom. The objective of this article is to take into consideration different views on this topic, with a view to reflect and consider different positions that allow the approach of a pedagogical strategy based on the art of dance, with which it is possible to visualize a broader perception that sets in motion a new approach to inclusion in the classroom, it is concluded, that dance is a fundamental element that tends to the improvement of interpersonal relationships integrating all educational agents that include children with disabilities or not as well as their caregivers, by allowing the recognition, empathy of self and acceptance of the other. without any type of discrimination.

\section{KEYWORDS:}

Inclusive education - dance, caregivers

\section{INTRODUCCIÓN}

La discapacidad ha sido ampliamente discutida a lo largo de los años, dando lugar a diferentes posturas y conceptos reflejados en modelos educativos que pretenden ser implementados con este tipo de población. En un comienzo, era discriminatorio y excluyente, puesto que pensó que tenían necesidades diferentes y como tal su escuela también debía serlo; posteriormente, fueron tenidas en cuenta y se consideró que deban ser integrados al sistema educativo común y no insertos en una educación especial y sectorizada.

Es fácil comprender entonces, como poco a poco han sido eliminadas las barreras restrictivas impuestas a este tipo de personas, para dar paso a la educación inclusiva, donde las personas con discapacidad se relacionan sin ningún tipo de segregación, en el contexto de una educación formal.

Ahora bien, la política de educación inclusiva en Colombia establece algunas estrategias pedagógicas y complementarias que plantean la necesidad de implementar ajustes razonables y flexibles en los currículos académicos, para promover el uso de herramientas y recursos didácticos incluyentes, para lo cual ha dispuesto del apoyo pedagógico de profesionales especializados, determinando a su vez la exigencia de una infraestructura accesible en los centros educativos, todo ello para lograr la implementación de esta política en el contexto de la educación formal (Ministerio de educacion y Gobierno de Colombia, 2017) (Decreto 1421, 2017).

Estas premisas teórico-conceptuales exigen a su vez la implementación de unas concepciones éticas que conciban la inclusión como un punto de derechos y de valores, lo que significa implementar estrategias de enseñanza distintas a las tradicionales, que dando así apertura a una educación que reconozca los diferentes estilos 
de aprendizaje, ofreciendo alternativas de acceso al conocimiento y a la implementación de competencias inclusivas dentro del aula de clase.

Conforme a lo anterior vale la pena resaltar, que el sistema educativo común se enfoca y prioriza la enseñanza de conocimientos relacionados con las ciencias, reconociendo este campo como fundamental en el desarrollo cognitivo de los niños y niñas, sin brindar la suficiente importancia al campo de las artes, las cuales aportan de forma significativa al desarrollo de los estudiantes, desde una perspectiva intelectual, física, social, expresiva y afectiva (Nicolás et al, 2010).

A partir de este marco de referencia, se propone que el arte propicie una deconstrucción y regeneración de nuevas dinámicas de enseñanza, que den cabida a la danza como un ejercicio significativo en la educación inclusiva, impulsador de relaciones interpersonales entre estudiantes con y sin discapacidad, en un mismo espacio académico, donde se puedan ver reflejadas diversas competencias que aporten a la formación de los niños y niñas desde una perspectiva integral, cuya base fundamental es el desarrollo socio emocional, comunicativo, comportamental, ciudadano, cultural y artístico.

Dicho de otro modo, la expresión danzaría se presenta como un medio pedagógico, para lograr la inclusión y aceptación de los estudiantes con discapacidad en la escuela formal y así, visibilizar esa transversalidad ética desde el campo de las artes, proyectando un espacio académico dirigido a trabajar en el respeto, el reconocimiento del otro, la empatía, la tolerancia, la solidaridad, la comunicación asertiva $y$ todos aquellos aspectos que competen a las sanas relaciones sociales, como seres humanos a la hora de interactuar con nuestro entorno a través del cuerpo.
Esto conduce a afirmar como lo puntean Acosta, L., Lugo, J., \& Solano, F. (2017) que la escuela necesita estar involucrada en un proceso de innovación de sus prácticas educativas, para no seguir excluyendo a las personas con discapacidad, promoviéndola desde la educación primaria, por ser la base fundamental en la construcción del comportamiento, conceptos, personalidad y carácter de los niños y niñas, de tal manera que se formen para ser jóvenes o adultos con capacidad de aceptación de la diferencia, basada en el desarrollo de una autoconciencia individual que fortalezca la conciencia colectiva, basada en el principio de la convivencia ciudadana.

La reflexión en curso se realiza desde un abordaje cualitativo, usando como herramientas de construcción de datos el análisis de documentos sobre el tópico de la inclusión, que será formulada como base para impulsar la propuesta planteada por quienes hacen este escrito. A continuación, se detallan algunos fundamentos teóricos, contemplados en este artículo.

\section{EXCLUSIÓN- INCLUSIÓN}

La discapacidad ha dado apertura a la inclusión y la exclusión hasta llegar a convertirse en ejes fundamentales en cualquier proyecto educativo, razón por la cual incluso las entidades gubernamentales en este caso, tanto los estamentos que lideran las políticas educativas, hasta los entes políticos (alcaldías, gobernaciones y otras entidades del Estado), han generado diversas normas que buscan responder a las falencias halladas a su alrededor.

Para dar claridad a cada uno de estos términos, es necesario establecer su definición, Parrilla (2002), especifica que la inclusión se aplica a múltiples contextos, indicando que no solamente se ubica en el educativo, por lo cual transversaliza grupos tales como la familia, las comunidades y los contextos sociales; 
Por otro lado, Sierra (2000), plantea que la inclusión abarca la diversidad, en términos de atención a la diferencia, dada por la condición individual de cada ser que lo lleva a interactuar dentro de una sociedad, caracterizado como un ser único, con necesidades, intereses y tipologías distintas, haciéndolo un ser disímil y singular, razón por la cual, espacios como la escuela deben evitar promover la homogeneidad en los procesos de aprendizaje y por el contrario, debe atender a esa multiplicidad de características propias, en cada uno de sus integrantes.

Con las premisas hasta aquí explicadas, se evidencia que no existe un concepto universal del término de inclusión, más bien, sirve para identificar diversas situaciones que atañen a múltiples individuos en los contextos donde se encuentran inmersos, sin embargo, no se puede dejar de lado algunas características asociadas a la diversidad, la individualidad del ser, que lo hace distinto a los demás, aunque se encuentre inmerso dentro de un grupo, por su sentido de unicidad.

Desde esta perspectiva se puede tomar en consideración elementos como la existencia, la adquisición del conocimiento y el compromiso, por ser fundamentales dentro de cualquier proceso de inmersión y aceptación, que hace parte del contexto de la inclusión, impidiendo usar cualquier tipo de obstáculo que imposibilite las relaciones de interacción entre los individuos pertenecientes a una comunidad.

Se abordará ahora la exclusión, para su presentación vale la pena traer a Estivill (2003) quien explica las diversas esferas que involucran este tema, cuyas repercusiones se basan en las múltiples rupturas que afectan tanto a la economía, la política y la sociedad, alejando a las personas y a los grupos de las oportunidades brindadas por el Estado, hasta llegar casi a invisibilizarlos.
Lo relevante de esta cuestión es la imposibilidad de incorporar a estas personas dentro de un círculo social, especialmente por el señalamiento de inferioridad resaltada por este autor, definición que lo conduce a ser concebido en términos de desventaja, por lo cual podrían correr el riesgo de ser aislado, perdiendo así sus derechos ciudadanos, enajenándose de este modo, de toda posibilidad de inserción en un núcleo social.

Esta imbricación de señalamientos permite determinar al mismo tiempo que Estivill (2003), amplía su mirada para referirse a la segregación que conduce a un patrón de comportamiento, dentro del cual las personas con discapacidad conviven separadas de la comunidad que los circunda, condición que los lleva a ser tratados de manera diferente y con miramientos especiales que subvaloran sus capacidades, anulando así sus posibilidades de integración en un colectivo, corriendo el riesgo de llevarlos a ser parte de un grupo minoritario, para finalmente resultar siendo excluidos, del resto de la sociedad.

Una vez contextualizada esta situación, se comprende la existencia de instituciones que cumplen la función de atender las necesidades de estos sujetos, mal llamados "especiales", se añade a esto que, han sido creadas con el propósito de suplir las carencias de este tipo de población, catalogándolos en muchos casos como, "un grupo diverso".

Esta disertación también conduce a reconocer que en el campo educativo esta diferenciación ha sido ampliamente marcada, clasificando a los estudiantes en dos grupos, aquellos que gozan de todas sus capacidades y que comúnmente asisten a las escuelas tradicionales, y por el otro lado, están quienes tienen cualquier tipo de limitación física, mental o cognitiva, quienes por tener esa condición requieren del apoyo de un personal especializado, situación que ahonda aún más, esta condición de segregación. 
Referida esta temática, cobra importancia la integración, por enfatizar la unión de las partes en un todo, para este caso particular los grupos minoritarios, es decir, aquellos que tengan discapacidad requieren ser reconocidos y aceptados sin ningún tipo de segregación, de tal manera que sean incorporados dentro de una estructura social.

Adviértase aquí que, desde la mirada de los derechos humanos, la inclusión ha resultado ser una inquietud universal, cuyo propósito fundamental es evitar la discriminación, presentando alternativas respecto a las desigualdades especialmente en el campo educativo.

A pesar de los esfuerzos hechos desde las políticas nacionales e internacionales, aún se evidencia la existencia de prácticas sectorizadas que promueven la segregación en acciones del diario vivir, al interior de la sociedad, situación que trae como consecuencia, que algunos grupos especialmente de discapacitados, se sientan excluidos de su núcleo social, por las nulas o pocas oportunidades que se les brinda para incluirlos en el ejercicio laboral, social, económico e inclusive político, a tal punto, que en algunos casos se les obstaculiza su posibilidad de participación ciudadana en todo contexto social.

Este escenario determina que el proceso de inclusión ha venido avanzando de manera lenta, pero permanente, hasta llegar a convertirse en una bandera de entidades como la Unesco, las Naciones Unidas y muchos otros entes, tanto nacionales como internacionales, que se han preocupado por la promulgación de planes y proyectos, enmarcados dentro de las leyes que buscan propender por la inclusión, pero que en la realidad se ven opacadas por la ínfima inversión de recursos que propendan por este tipo de iniciativas y propuestas, que buscan democratizar la inclusión y la participación a nivel nacional y global.

\section{UNA MIRADA A LA EXCLUSIÓN EN EL CONTEXTO EDUCATIVO}

A pesar de los variados esfuerzos a nivel nacional e internacional por parte del Estado para generar políticas de inclusión, aún sigue siendo muy incipientes su implementación y sostenibilidad.

En este punto de la discusión, se puede traer a acotación a la Unesco (2008) por haber reconocido los reparos que se han dado respecto a las necesidades educativas detectadas en grupos poblacionales con necesidades especiales, frente a las cuales las políticas del estado muchas veces han promovido medidas provisionales, incompletas y fragmentarias, alejadas de la realidad de este tipo de población.

Bajo esta perspectiva se afirma que el incremento en la cantidad de escuelas especiales con currículos diferentes y docentes de educación especial no se deben tomar como sinónimo de inclusión, aunque es cierto que, esta propuesta logró incorporar a las personas con discapacidad dentro del sistema educativo, sigue siendo una forma de excluir $y$, por lo tanto, de segregar dentro de la educación a esta población (UNESCO, 2008).

A este propósito, Vaillant (2011) explica que en Latinoamérica la educación inclusiva ha pretendido resolver el tema del estatus de este tipo de población y la carencia de docentes capacitados para afrontar este tópico profesionalmente, razón por la cual ha sido indispensable el planteando de unos lineamientos educativos relacionados con este campo.

En respuesta a ello, Calvo (2013) cree ineludible que los procesos educativos se desarrollen bajo los principios de igualdad y equidad, haciendo énfasis en la necesidad de promover una capacitación integral en las instituciones formadoras de docente, que 
eduque profesionales con los cuales se dé respuesta a las múltiples necesidades de este tipo de ciudadanos, de tal manera que sus requerimientos se vean reflejados tanto en las políticas, como en los currículos desarrollados en las diversas entidades escolares, con las cuales se fortalezca la educación inclusiva.

\section{POLÍTICAS DE INCLUSIÓN EN EL CONTEXTO COLOMBIANO}

En lo que respecta a la política Colombiana relacionada con la inclusión son múltiples los autores que se han preocupado por abordarla, entre ellos se encuentran Pérez (2016), Canal (2014), Hernández y Velázquez (2016) y Agudelo M y Hurtado (2016), quienes plantean que desde la constitución política de 1991 se empezaron a generar lineamientos relacionados con este tipo de pobladores, de igual manera, se han emanado leyes como la 115 de 1994 , la cual resume la normatividad colombiana teniendo como referente la Constitución Política de 1991, las leyes 115 de 1994, 361 de 1997, 1098 de 20061145 de 2007, 1346 del 2008, 1346 de 2009, Auto 006 de 2009, el documento CONPES 80, el Plan Nacional de Desarrollo y el Plan Nacional de Intervención en Discapacidad, que le otorgan al Estado la responsabilidad de responder por la educación inclusiva de calidad a los colombianos, promulgando una educación que no dé cabida a la diferencia.

Todos estos lineamientos han sido canalizados por diversas entidades gubernamentales, las cuales a través de algunas de sus normativas pretenden suscitar la educación inclusiva, para dar respuesta a una de las causas de la deserción escolar y así atender la retención escolar.

Para cerrar, es pertinente agregar que este tipo de políticas se basan en el acceso, gestión y seguimiento a los programas, con los cuales se encausen acciones benéficas para la población de discapacitados.
Continuando con la mirada política, es necesario recalcar que a mediados de los años 80 y 90 se empezó a implementar el concepto de integración, como respuesta a la problemática de segregación dentro de este tipo de población, la cual considera que los estudiantes con discapacidad puedan asistir a las escuelas comunes (UNESCO, 2008), desmontándose así las pocas escuelas especiales que existían en el contexto colombiano.

En respuesta a esto, se propone que estos estudiantes se incorporen al sistema educativo tradicional, haciendo pequeñas variaciones a los currículos, basados en el diagnóstico del estudiante con discapacidad, pero no respondiendo a fondo a la diversidad de necesidades de estos educandos.

Es interesante advertir cómo la integración dispone de un traslado espacial de la educación especial dentro de la educación formal, es decir, estos jóvenes asisten a la escuela con todos los demás niños y niñas sin exclusión, ni distingo alguno, pero dentro de estas escuelas se realizan clases para todos los estudiantes, sin contemplar los atenuantes de su discapacidad, esto especialmente por la falta de capacitación docente y a la escasez de personal especializado e interdisciplinar, que brinde un acompañamiento permanente al docente, para atender a este tipo de población, por lo tanto, la inserción es parcial, condicionada y simulada, trayendo además conflictos tanto para el profesor, como para el estudiante, sus familiares, acompañantes o cuidadores, quienes se sienten frustrados al no poder atender, ni ser atendidas sus necesidades particulares.

En resumidas cuentas, se puede apreciar que las políticas del Estado en lo relacionado con el campo de la inclusión han tratado de dar respuesta a esta problemática, pero de manera muy somera y lenta, sin realmente apuntar a las dificultades de fondo que puede tener este tipo 
de población, quedándose muchas veces cortos en sus alcances, especialmente en el campo de las oportunidades, ya sea de tipo laboral, profesional o educativo, o a nivel superior, de allí la pertinencia de esta propuesta danzaria.

El tejido de decisiones metodológicas a ser atendido en una actividad de danza en el aula, nos lleva a involucrar a Arnaiz (2003) por promover principios aplicados en un contexto de educación inclusiva, en primer lugar, precisa que las clases deben estar enmarcadas en la diversidad contemplando las características individuales de los participantes de las clases, de esta manera, se tendrá en cuenta la unicidad que en conjunto debe alcanzar la integralidad, adoptando la inclusividad como elemento fundamental, para dar viabilidad a este compendio es necesario tener en cuenta la flexibilidad en el currículo que propicie el trabajo colaborativo, basado en una interacción y cooperación con un grupo de personas que buscan alcanzar un propósito común, el cual para este artículo, será la motivación para la organización de una presentación de danza; para alcanzar este propósito es importante la formación del maestro, en el momento en que decida implementar una estrategia metodológica para desarrollar la temática específica, que en este caso se relaciona en la danza.

Según Arnaiz (2003) es fundamental que los estudiantes se vean involucrados en un problema dentro del cual ellos estén comprometidos, proponiendo diversas soluciones, viables y alcanzables acordes a las condiciones dentro de las cuales se encuentran inmersos los participantes de la propuesta danzaría para este caso, en este punto agrega el autor, se requiere de una valoración frente a la actividad desarrollada, de tal manera que se tome en cuenta su opinión y su pensamiento crítico, hasta llevarlo a ser protagonista del proceso dentro del cual él y su familia, están involucrados.
Es de resaltar que en este tipo de iniciativas educativas se rompe con los cánones tradicionales de enseñanza - aprendizaje, para dar cabida a dinámicas interactivas que incluyen todo tipo de población e interacción.

Una vez presentados estos miramientos, se procede a explicar otras premisas de este autor, quien enfatiza la necesidad de contar con un apoyo permanente a los profesores que acompañan esta iniciativa de enseñanza, contemplando un contexto inclusivo, para lo cual es menester reconocer que requieren de un apoyo económico, social y psicoafectivo, que involucre tanto a padres y familiares como a ellos mismos, promoviendo de esta manera los aspectos culturales inherentes a su idiosincrasia, para ofrecer una educación de calidad que reconozca el derecho de la inclusión, erradicadora de todo principio de discriminación.

Con todo esto, cabe resaltar la importancia del acompañamiento de los padres como fundamento de inclusión, por convertirse en un factor determinante para impulsar todo tipo de iniciativas y más aún aquellas que permiten el desarrollo de la danza, como parte esencial de una escuela flexible, posibilitadora de espacios de enseñanza que dé cabida a la participación de todos, sin tener ningún tipo de discriminación por su condición socioeconómica, cognitiva, expresiva o familiar, sino por el contrario, que sea impulsadora de una inclusión educativa en un contexto curricular, promotor de ideas innovadoras y participativas en pos de la comunidad.

Hasta aquí, se han expuesto enunciados fundamentales promotores de una escuela inclusiva, ahora daremos paso a la presentación de algunas estrategias que son determinantes para viabilizarlos y visibilizarlos, tomando la mirada de Arnaiz (2003), se reconoce como punto inicial, la activación de una escuela heterogénea cimentada en el alcance de unos objetivos, que han sido compartidos y acordados 
por un equipo de expertos, dentro del cual se han tomado decisiones en comunidad, definiendo unas metas a corto y mediano plazo que sean alcanzables, basados en su compromiso.

Como segundo aspecto, se recomienda trazar metas a largo plazo que busquen involucrarlos a todos, de tal manera que se contemplen sus expectativas y estimaciones, con el propósito de motivar acciones conjuntas que satisfagan las necesidades personales y grupales; esta apreciación se amplía en el tercer componente que hace referencia a la participación de quienes integran y acompañan la escuela, para dar cabida tanto a los padres, las directivas, los estudiantes, así como al resto de personas (cuidadores, acompañantes, familiares 0 padres de familia) que se relacionan con estos niños, esto para propiciar una conversación comunicante y a su vez de responsabilidad, por el compromiso entre quienes conforman la escuela y buscan un único objetivo, como es el de promover la inclusión de sus hijos, en la sociedad.

En tercer término, es crucial el rol desempeñado por los profesores especializados en la educación inclusiva quienes tienen la responsabilidad de encausar una enseñanza significativa y creativa, basada en el desarrollo de las destrezas sociales $y$ afectivas, que potencializan las habilidades y aptitudes de cada uno de los participantes del aula.

En cuarto lugar, se requiere de un docente comprometido con esta causa, convencido de la necesidad de promulgar una enseñanza incluyente que propenda por la flexibilidad de la escuela, para dar cabida a propuestas alentadoras de las capacidades tanto cognitivas, afectivas, así como expresivas en los niños y niñas, alejadas de los procesos de masificación y que por el contrario, tengan en cuenta las posibilidades, los intereses, las individualidades y las caracterizaciones de cada individuo, que hace parte de la escuela, para enriquecer el todo, es decir, la ciudadanía.

Cabe decir entonces, como lo señalan Agudelo, M., \& Hurtado, L. (2014) que este docente debe interactuar permanentemente con los otros colegas para suscitar el diálogo, la reflexión y el deseo de compromiso, contribución y apoyo a la comunidad, encaminando sus acciones en proyectos colectivos, involucradores y participativos como la organización de una presentación danzaría.

Por último, se observa que la promoción de centros dedicados a la educación especial, indirectamente fueron focos de discriminación, donde las limitaciones fueron importantes, más no las posibilidades y potencialidades que tenían los individuos al ser integrados en una comunidad más amplia, la cual indirectamente podía aportarle al desarrollo de su capacidad y destreza.

\section{PROPUESTA MODELO BIODANZA INCLUYENTE}

La sociedad desde la época primitiva ha asumido la danza como elemento fundamental de su cultura, hasta convertirla en una forma de expresión no verbal que entrelaza los saberes, las emociones y las creencias, llegando a ser cristalizada en ritos y/o mitos que la trascienden, generando nuevas formas de manifestación de creencias, sentires, necesidades y vivencias, que con el pasar del tiempo perpetúan la cultura y las tradiciones de los pueblos.

Aquí vale la pena reconocer como lo plantea Nicolás (2010), que la danza es más que un movimiento impulsivo del cuerpo, que trasciende en la medida que sus participantes le imponen la energía, la espiritualidad y la emoción para convertirse en una expresión del estado de ánimo de un colectivo, dejando ver una armonía y un sentido estético grupal, transformándose en una oportunidad para aquellas comunidades 
discapacitadas invisibilizadas por mucho tiempo, en el campo educativo y en la sociedad en general.

Ahora es oportuno hacer una reflexión frente a las ventajas que se pueden descubrir durante la organización de una presentación de danza por ser incluyente, integral, colaborativa y participativa de todas aquellas personas interesadas, en sacar adelante este propósito.

\section{EL ARTE DANZARIO COMO ELEMENTO DE INCLUSIÓN}

Tomando como referencia la política Colombiana frente a la educación inclusiva determinada por el decreto 1421 de 2017, el cual estipula la implementación de diversas estrategias pedagógicas, formativas y complementarias, esta propuesta va encaminada a crear espacios asertivos organizador de relaciones interpersonales, entre un grupo de estudiantes de niños o niñas con discapacidad o no, así como a sus cuidadores o progenitores, quienes a través de la planeación de la presentación artística, los comprometa e integra.

De ahí la importancia de examinar el arte de la danza como eje fundamental para este tipo de población, tomando el cuerpo como una herramienta facilitadora y eficaz de la comunicación e interacción con el entorno.

Ahondando un poco más frente a los beneficios de la danza y sus aportes en la dimensión social y la interconexión con los otros, se reconoce su impacto en el espacio socio afectivo, por ser una herramienta facilitadora del trabajo colectivo, expresivo, receptivo y potencializador de habilidades cuidadoras, que promuevan el contacto físico y corporal con los demás, permitiendo a su vez el autorreconocimiento corporal y la apreciación del otro como un igual, así como la comunicación no verbal, como lo señala (Nicolás et al, 2010), situación que fomenta la unificación participativa y social dentro y fuera del aula, especialmente con este tipo de población. Ahora se dará una explicación respecto a su implementación.

Esta propuesta ha trazado como principal objetivo generar escenarios de creación artística, expresión de emociones y trabajo solidario, a través de la danza.

Por otro lado, se pretende proponer un modelo de planeación danzaría incluyente, que permita la participación de los cuidadores, así como de los niños y niñas con discapacidad, donde también confluyan sus diversos compañeros que también hacen parte del aula escolar.

\section{METODOLOGÍA}

Para llevar a cabo esta propuesta se organizaron diversos talleres relacionados con temáticas que exploraron sus gustos musicales, sus costumbres y sus lugares de origen, mediante los cuales se pudo reflexionar acerca de la importancia del reconocimiento de la cultura, enfatizando la interacción con el otro, asignando diferentes responsabilidades compromisos y roles.

En este sentido, los ejercicios desarrollados respondieron a una metodología participativa y experiencial, propiciadora de lazos de confianza y acercamiento, es por ello, que se recomienda que el educador que lidere este tipo de actividades debe contar con un conocimiento profesional sobre la danza, un alto entusiasmo y un gran sentido de liderazgo, para sostener la interacción respetuosa entre pares, fomentando un ambiente empático y de construcción, tanto cognitiva como corporal y emotiva, desde la dimensión individual, colectiva e integrada.

En el siguiente gráfico se ilustra la propuesta en un modelo. 


\section{MODELO DANZARIO INCLUYENTE}

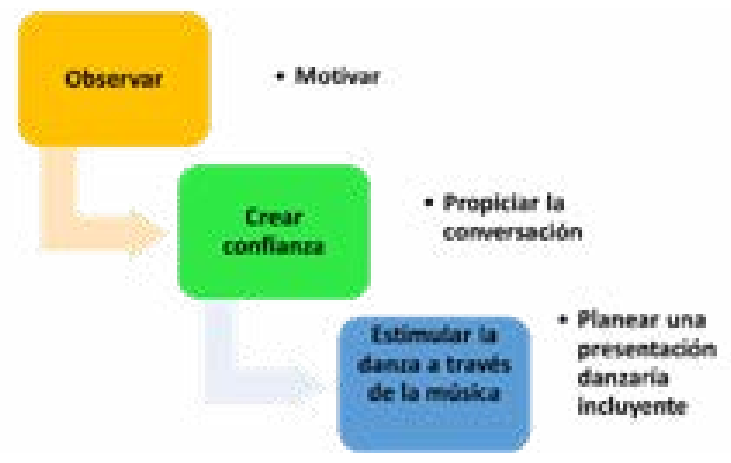

Grafica 1. Propuesta de actividades a ser implementadas. Elaboración propia.

Con este modelo se busca subsanar la brecha entre lo invisible y lo incluyente en un grupo de niños con discapacidad, a través del arte en este caso la danza, en un tiempo relativamente corto. Ahora se explica, algunos de los pasos contemplados.

\section{OBSERVAR}

Se sugiere hacer una actividad que promueva el diálogo continuo y enriquecedor entre los participantes (niños y niñas, estudiantes, cuidadores, padres, y toda aquella persona que sea vinculante en la actividad de la danza), esta estrategia busca propiciar un ambiente de adaptación entre los participantes, pero a su vez brinda información sobre el tipo de actividad que pueda ser desarrollada en esta clase de talleres, de allí la importancia de prestar atención a cada detalle que se pueda ver, para así determinar el tipo de melodía, música y ritmo, que se ajuste al grupo y así poder organizar la presentación danzaria.

Relación y contacto. En este ítem, los ejercicios de la exploración del cuerpo y del movimiento propuestos por el docente, deben buscar que los niños tomen conciencia de la relación con su cuerpo y con de otros, resaltando el cuidado y el respeto.
Se advierte aquí que el contacto físico es una buena herramienta para generar relaciones de mayor confianza, pero es necesario evaluar en cuales momentos puede establecerse ejercicios de contacto y en cuáles no, priorizando la comodidad de cada niño.

\section{Comunicación corporal.}

En este momento del taller se recomienda organizar actividades en parejas o pequeños grupos, propiciadoras de una comunicación no verbal entre ellos, por medio de juegos que promuevan la exteriorización de sentimientos, valores, emociones y pensamientos, a través del gesto facial y corporal para romper el hielo entre los participantes, dando lugar a sus diferencias, es decir, es importante establecer una interacción vinculante entre iguales.

\section{Crear confianza}

Basados en los ejercicios de interacción que ya se han debido propiciar en la primera parte del taller, es necesario entrar a planear otras actividades que promuevan la confianza, la familiaridad y la fraternidad, entre otros, detalles que son de suma importancia para generar lazos de amistad y compañerismo, entre los participantes.

\section{Rol del guía.}

Mas que un instructor o un profesor, el docente orientador y organizador de la presentación de la danza tendrá que ser un guía, que motive las experiencias corporales de cada niño dentro de su diversidad, sus capacidades y su comodidad. Igualmente, es indispensable suscitar un ambiente abierto a la escucha, empático, tranquilo y seguro, que garantice la confianza en los niños y atenúe la expresión de cualquier inquietud, incomodidad, pensamiento, o sugerencia, de igual modo, se promover relaciones entre pares de forma ética, que incluya a los cuidadores, o a los padres; por lo cual, es importante, sostenerse que se debe 
prestar atención y respetar las necesidades de cada niño y de todos los participantes que harán parte de la presentación.

En lo posible, para la implementación de un espacio de danza incluyente se debe contar con un guía adicional o de apoyo dentro del espacio de la escuela. Cabe resaltar, que no se trata de una clase de danza dirigida a la imitación de movimientos propuestos por el profesor, o a la réplica de una coreografía, sino que se dirige a una exploración del cuerpo y de las posibilidades del movimiento de cada uno y desde la sinergia grupal, esto es, en pro de evitar frustraciones, al no poder ejecutar un movimiento propuesto de forma técnica, en ese sentido, cada uno explora desde las posibilidades de su ser, no solo físicas, sino también de imaginación, de ideas, y de creación, por lo tanto, la disposición del guía debe estar abierta a los cambios o variaciones que surjan en el proceso, se sugiere entonces ser flexible y relajado, sin condicionar u obligar a que todos participen en todas las actividades, ya que los niños pueden tener diferentes formas de intervenir y de sentirse cómodos, al igual que sus cuidadores o padres.

Finalmente, la danza se concibe como un medio para lograr la inclusión en el aula, si se tienen en cuenta ciertas pautas en su implementación, las cuales corresponden a alguno de los elementos que a continuación se explican:

\section{Rol estudiante.}

Es circunstancial, en un primer momento, es necesario comunicarles a los niños y niñas participantes ciertas consideraciones a ser tenidas en cuenta para la organización de la actividad, esto para establecer acuerdos de convivencia mínimos, dentro del espacio donde se desarrollarán los diversos talleres y encuentros, por lo cual deberán ser enfocados hacia el respeto, la empatía y la responsabilidad en el manejo de su cuerpo y en la del compañero.
Al establecer estas reflexiones, es necesario plantear que la inclusión no es solamente una responsabilidad de los educadores, sino también es un deber de los niños y niñas que serán copartícipes de la actividad danzaría, esto porque tienen derecho a manifestar cualquier duda, inquietud, u opinión. En este sentido, ellos deben tener una reflexión consciente y constante, durante cada sesión del taller, de tal manera que se organicen espacios de reflexión sobre la experiencia vivida, donde se expresen las sensaciones, emociones y pensamientos frente a los ejercicios corporales, llevados a cabo junto a sus compañeros, cuidadores o padres de familia, de esta manera, se tendrá una aproximación cualitativa al proceso continuo, flexible y acumulativo con una orientación de emotividad, que hará la diferencia de la escuela tradicional.

\section{Estimular la danza a través de la música.}

La exploración y expresión corporal son aspectos de suma importancia en la implementación de una propuesta danzaría en un contexto incluyente, por lo tanto, se constituye en un elemento de éxito en la organización de este tipo de apuestas donde los niños y las niñas deben sentirse cómodos con su entorno, para alcanzar ese propósito, se sugiere la implementación de ejercicios de respiración, de calentamiento, de meditación y espaciales, con los cuales se tenga una identificación de las locaciones a ser utilizadas para el montaje de la presentación de la danza, de esta forma alcanzarán un alto grado de confianza, cuando estén en el escenario.

Las actividades deben incluir elementos corporales de reconocimiento de movimiento dentro de las cuales es muy importante el juego, el tiempo, el ritmo musical y el espacio. Es significativo que se propenda por integrar las diferencias convirtiéndolas en oportunidades de enriquecimiento del proceso de aprendizaje en conjunto, de tal manera que se subsane toda deficiencia u obstáculo por más mínimo que sea; 
de esta forma se pretende que los integrantes que harán parte de la presentación, reconozcan y descubran la diversidad corporal de cada uno de ellos, porque a pesar que posean diversas alturas, contexturas, destrezas y habilidades, lo más transcendental es destacar sus capacidades, identificando las potencialidades que enriquecen el trabajo colectivo y ético del grupo.

Creación Inclusiva: Las actividades incluidas en esta etapa final se enfocarán en la coordinación de movimientos armónicos establecidos en conjunto, en los juegos de improvisación grupal e individual que aporten a la imaginación del movimiento, que pueden serán visualizados al ponerse en escena como en una presentación de cierre resultado de un producto colectivo.

\section{CONCLUSIONES}

En el campo de la educación para la población con discapacidad han sido poco notorios los cambios a lo largo de los años, pero hace corto tiempo, se ha venido observando una amplia acogida en los centros educativos formales, que han dispuesto apuestas por la inclusión, como producto de la reglamentación estatal, de la cual se espera que, a largo plazo, se vea reflejada en las diversas acciones enmarcadas en las oportunidades sociales y de calidad para esta población, ciertamente, en la normativa se han determinado diversas políticas relacionadas con la educación inclusiva, sin embargo, es menester reconocer que aún falta el emprendimiento de acciones prácticas que reflejen en la realidad, la puesta en marcha de actividades que encaminen el liderazgo y la implementación de cambios, que evidencien nuevas acciones en términos de inclusión.

De aquí se desprende la necesidad de hacer conciencia en los docentes frente al liderazgo y la responsabilidad que tienen en el campo de la inclusión, para buscar un equilibrio con su grupo de trabajo, poniendo en marcha propuestas como las que aquí se plantean, que propenden por la implementación de las políticas de inclusión, que a pesar de ser muchas, son pocas las que se tienen en cuanto a su ejecución.

Desde la perspectiva de la educación para todos y todas, es preciso reconocer que la danza toma y reivindica la educación inclusiva, por sus múltiples aportes y alcances tanto en lo cognitivo, lo físico motriz, lo emocional y lo bioético, así como en el desarrollo humano, suscitando al mismo tiempo el desarrollo integral, socio emocionales y afectivo que propenda por una verdadera educación incluyente.

En este sentido, la danza permite el fortalecimiento de las cualidades personales e interpersonales generando nuevas dinámicas sociales, que posibilitan la percepción de nuestro entorno que median en el tejido social, cognitivo, así como en la actitud de la sociedad de nuestro tiempo, hacia este tipo de población, tal como lo reconoce Quintana (2019), razón por la cual se sugiere ser consideradas las sugerencias aquí planteadas, en toda apuesta promotora de la educación inclusiva.

\section{BIBLIOGRAFIA}

Acosta, L. L. (2017). Educación inclusiva en las instituciones educativas de Caicedonia Valle del Cauca. Prospectiva.

Agudelo, M. \&. (2014). Inclusión educativa de las personas con discapacidad en Colombia. Movimiento y salud. Obtenido de http://riberdis.cedd. net/ handle/11181/4889

Arnaiz Sánchez, P. ( 2003). Educación Inclusiva: una escuela para todos. Argentina: Ed. Aljibe.

Canal, J. (2014). Discapacidades en la escuelA. Revista de Investigación Silogismo. 
Colombia, C. d. (1993). Ley 60/1993 de 12 de agosto. Congreso de Colombia. Colombia.

Colombia, C. d. (1994) ). Ley 115/1994. Congreso de Colombia. Colombia .

Colombia, C. d. (1997). Ley 361/1997 de 7 de febrero. LCongreso de Colombia. Colombia .

Colombia, M. d. ( 2017). Decreto 1421 de agosto 29 de 2017. Ministerio de Educación y Gobierno de Colombia.

Colombia., C. d. ( 2006). Código de la Infancia y la Adolescencia. . Congreso de la República de Colombia. (2006). Ley 1098 de 2006. Código de la InDiario Oficial N 46.446. Bogotá, D.C, .

Congreso de la República. Ley 1346 de 2002 [Internet]. [citado 27 de septiembre de 2013]. Recuperado a partir de: http://www.secretariasenado.gov.co/ senado/basedoc/ley/2009/ley_1346_20 09.html).

Congreso de la República. Ley 1346 de 2002 [Internet]. [citado 27 de septiembre de 2013]. Recuperado a partir de: http:// www.secretariasenado.gov.co/senado/ basedoc/ley/2009/ley_1346_20 09.html.

Constitucional, C. (2009). Auto 006 de 2009: sobre el impacto cualitativamente diferenciado y agravado sobre las personas con discapacidad. . Corte Constitucional.

Constitucional, C. (Corte Constitucional. Auto 006 de 2009: sobre el impacto cualitativamente diferenciado $y$ agravado sobre las personas con discapacidad)
Estivill, J. (2003). Panorama de la Lucha Contra la Exclusion. Conceptos y Estrategias. Ginebra. Oficina Internacional del Trabajo. Obtenido de http://www. derechoshumanos.unlp.edu.ar/assets/ files/documentos/panorama-de-lalucha-contra-la-exclusion-

Hernández, E. \&. (2016 ). Comprensiones del discurso normativo sobre inclusión educativa en Colombia. El Ágora U.S.B.

Ministerio de Educación y Gobierno de Colombia. ( 2017). Decreto 1421, 2017. Ministerio de Educación y Gobierno de Colombia.

Nicolás, G. V. (2010). La danza en el ámbito de educativo. Nuevas Tendencias en Educación Física, Deporte y Recreación. Obtenido de https://www.redalyc.org/ articulo.oa?id=345732283009

Pérez, L. (2016 ). Praxis inclusiva, para la atención de necesidades educativas especiales en el sector rural de Bucaramanga. Praxis \& saber. Obtenido de http://dx.doi.org/10.19053/22160159. v7.n15.2016.5726

Planeación, D. N. ( (2004 ). Conpes Social 80. Política Pública Nacional de Discapacidad. Bogotá, Julio.

Quintana, R. (2019 ). La Danza en las dimensiones del Ser Humano y los contenidos escolares. Plumilla Educativa.

Secretaria del Senado (2002). Ley 1346 de 2002 . secretaria senado.gov.co. Obtenido de http://www.secretariasenado.gov.co/ senado/basedoc/ley/2009/ley_1346_20 09.html

UNESCO. (2008). LA EDUCACION INCLUSIVA: EL CAMINO HACIA EL FUTURO. UNESCO. Ginebra: Unesco. 
Vaillant, D. (2011 ). Preparing teachers for inclusive education in Latin America. Prospects. Obtenido de http://dx.doi.org /10.1007/s11125-011-9196-4 